\title{
Sistemas de Saúde e Competências do Enfermeiro em Portugal
}

\author{
Health Systems and Nursing Skills in Portugal
}

Ana Paula da Silva Rocha Cantante (https://orcid.org/0000-0002-3839-344X) ${ }^{1}$

Henriqueta Ilda Verganista Martins Fernandes (https://orcid.org/0000-0002-8440-3936) ${ }^{1}$

Manuela Josefa Teixeira (http://orcid.org/0000-0002-2460-8796) ${ }^{1}$

Mirna Albuquerque Frota (http://orcid.org/0000-0003-3004-2554) ${ }^{2}$

Karla Maria Carneiro Rolim (http://orcid.org/0000-0002-7914-6939) ${ }^{2}$

Firmina Hermelinda Saldanha Albuquerque (http://orcid.org/0000-0002-0697-2789) ${ }^{3}$

${ }^{1}$ Escola Superior de Enfermagem do Porto. R. Dr. António Bernardino de Almeida 830. 4200072 Porto Portugal. apcantante@esenf.pt ${ }^{2}$ Universidade de Fortaleza. Fortaleza CE Brasil. ${ }^{3}$ Universidade Federal do Amazonas. Coari AM Brasil.

\begin{abstract}
The paper discusses the Portuguese Health System that has adopted the Beveridge model, which is based on the financing of health services by taxpayers' income, based on a public system, where the right to health is independent of work and employment. Nursing education is structured in Pre-Graduate Education - Degree; Master and Doctorate in Nursing Sciences and Nursing. The competency of the generalist nurses refers to the professional performance showing the effective application of knowledge and skills, which allows them to make a clinical judgment and decide. The exercise of competencies is based on the interpersonal relationship between the nurse and the individual client or group; decision-making based on scientific evidence, clinical judgment based on the needs of individual or group care, prescribed nursing interventions considering the safety of care and the client, early detection of the real or diagnostic potentials seeking resolution or minimization of consequences, by the values of the patients, as well as respect and professional regulation that establish good practice.
\end{abstract}

Key words Health Policy, Primary Health Care, Nursing
Resumo $O$ artigo discorre sobre o Sistema de Saúde em Portugal que possui o modelo de Beverigde, baseado no financiamento dos serviços de saúde pelas receitas, obtidas por impostos sobre o rendimento dos contribuintes, alicerçado num sistema público, em que o direito à saúde é independente do trabalho e do emprego. O ensino de Enfermagem está estruturado em: Formação Pré-Graduada - Licenciatura; Mestrado e Doutorado em Ciências de Enfermagem e Enfermagem. A competência do enfermeiro de cuidados gerais refere-se ao desempenho profissional demonstrador da aplicação efetiva do conhecimento e das capacidades, que the permitem o juizo clínico e a tomada de decisão. O exercício das competências baseia-se na relação interpessoal entre o enfermeiro e o cliente individual elou grupo; tomada de decisão baseada em evidência científica, juízo clínico fundamentado nas necessidades de cuidados individuais ou do grupo, intervenções de Enfermagem prescritas considerando a segurança dos cuidados e do cliente, detecção precoce dos reais ou potenciais diagnósticos buscando resolução ou minimização das consequências, pelos valores dos clientes, além do respeito e regulamentação profissional que estabelecem a boa prática.

Palavras-chave Políticas de Saúde, Atenção Primária à Saúde, Enfermagem 


\section{Introdução}

Portugal (continente e os dois arquipélagos) atualmente tem uma população total de 10,3 milhões de habitantes, concentrados, sobretudo nas áreas metropolitanas de Lisboa, do Porto e no litoral, ficando o interior do país mais desertificado ${ }^{1}$.

A partir de 1974 acentuou-se um notável desenvolvimento humano, social e econômico, sobretudo após a adesão à Comunidade Econômica Européia (1986) e à Zona Euro (1999) influenciando positivamente os determinantes da saúde. Concomitantemente assistimos a um aumento da esperança média de vida, passando as mulheres de 71,4 anos e os homens de 64,8 anos, em 1974, para 83,4 e 77,8 anos, respectivamente, em 2017, sendo coincidente com os valores da União Européia - UE (de 28 países em 2017)². As mulheres possuem maior longevidade do que os homens, porém são mais afetadas por patologias músculo-esqueléticas, depressão e obesidade ${ }^{3}$.

Melhores condições de vida, associadas a uma maior oferta de serviços de saúde de qualidade e à diminuição da prevalência de algumas doenças, contribuíram para prolongar a vida dos Portugueses. Isso resultou num envelhecimento demográfico, em que a esperança de vida aos 65 anos, em 2017, era de 19,5 anos, em média, para os dois sexos, sendo que existem 153,2 idosos por cada 100 jovens ${ }^{4}$.

Quanto ao acesso, o principal obstáculo são as disparidades geográficas que interferem na concretização das necessidades de cuidados médicos não satisfeitas, contudo semelhantes à média da UE. Desiguais são as despesas de saúde, ficando muito abaixo de outros países da UE. Portugal, em 2015, gastou 1.989 euros per capita em cuidados de saúde, cerca de $30 \%$ abaixo da média da UE (2.797 euros). Estas despesas equivalem a 9\% do PIB, sendo a média da UE de 9,9\%. O financiamento público cobre cerca de dois terços das despesas, contudo tem aumentado os pagamentos diretos recaindo nas despesas das famílias. Todavia os grupos vulneráveis estão isentos de copagamentos garantindo assim, a acessibilidade aos serviços de saúde ${ }^{5}$.

Portugal tem uma das mais baixas taxas de hospitalização evitável da UE e as taxas de mortalidade, sensível aos cuidados de saúde, embora com diferenças por sexo, estão em sintonia com as médias da UE4. Várias reformas têm sido feitas para reforçar a eficiência e a transparência do sistema de saúde, como a aplicação de medidas e políticas de saúde eficazes para a garantia da sustentabilidade financeira, essencial a qualquer sistema de saúde ${ }^{5}$.

\section{Sistema de Saúde em Portugal}

O conceito de Sistema de Saúde é abrangente. Para a Organização Mundial de Saúde (OMS) ${ }^{6}$ é a possibilidade de concretizar "todas as atividades que têm como finalidade essencial a promoção, a recuperação ou a manutenção de saúde" $(\mathrm{p} .40)$. Para Andrade ${ }^{7}$ é um "sistema funcional dirigido à prestação de cuidados de saúde, engloba o Serviço Nacional de Saúde - SNS e os estabelecimentos sob a alçada do Ministério da Saúde, apenas como mais um dos elementos, a par das entidades privadas e sociais (com ou sem fins lucrativos)" $^{\prime \prime}($ p.209).

Os sistemas de saúde no mundo podem ser pensados com base em questões específicas e na articulação de três componentes que nos vão conceder modelos distintos: Político (Modelo de Gestão), Econômico (Modelo e Financiamento) e Médico (Modelo Assistencial) ${ }^{8}$. Em Portugal foi instituido o modelo de Beverigde (inspirado no modelo do Reino Unido), que se baseia no financiamento dos serviços de saúde pelas receitas obtidas por impostos sobre o rendimento dos contribuintes, alicerçado num sistema público, em que o direito à saúde é independente do trabalho e do emprego.

Porém, o sistema fiscal progressivo em relação aos cuidados de saúde torna-se regressivo, pois tem uma significativa dependência dos impostos indiretos e sobrecarga no orçamento das famílias (que em 2016 correspondia a 27,8\%) e tem vindo a aumentar, para permitir a sustentabilidade financeira ${ }^{2}$.

Em 1990, em Portugal, surge a Lei de Bases da Saúde que apresenta um conceito de sistema de saúde que é "constituído pelo SNS e por todas as entidades públicas que desenvolvam atividade de promoção, prevenção e tratamento na área da saúde, bem como por todas as entidades privadas e por profissionais livres que acordem com a primeira na prestação de todas ou de algumas daquelas atividade", tendo como objetivo proteger a saúde da população que vive em Portugal ${ }^{9}$.

O sistema de saúde português, presentemente, integra uma combinação de financiamento tanto público como privado e como sistema funcional engloba: o SNS - financiado maioritariamente pelos impostos e os estabelecimentos, sob a jurisdição do Ministério da Saúde, entidades privadas e sociais com ou sem fins lucrativos. Existe, ainda, a aplicação de taxas moderadoras para consultas, 
serviços de urgência, visitas domiciliárias e meios complementares de diagnóstico e terapêutica, mas cerca de $60 \%$ da população está isenta do seu pagamento $^{4}$ (Figura 1).

A Entidade Reguladora da Saúde (ERS) é uma entidade pública independente, que tem por missão a regulação da atividade dos estabelecimentos prestadores de cuidados de saúde, criada pelo Decreto-Lei no 309/2003, de 10 de dezembro. Em 2016 foi criado o Conselho Nacional de Saúde, órgão independente, que reúne os principais atores do setor de saúde, procede como órgão consultivo do governo na obtenção de consensos, assim como produz estudos e recomendações sobre questões relacionadas com as políticas de saúde. As unidades de saúde privadas e as farmácias não estão integradas no SNS.

O Ministério da Saúde é o departamento governamental que tem por missão definir e conduzir a Política Nacional de Saúde, garantindo uma aplicação e utilização sustentáveis dos recursos e a avaliação dos seus resultados. Deve regulamentar, financiar, orientar acompanhar, avaliar, auditar e inspecionar o $\mathrm{SNS}^{10}$ (Figura 2).

O Ministério da Saúde tem hierarquicamente a administração direta dos serviços: SecretariaGeral da Saúde (SG), que assegura apoio técnico e administrativo aos restantes gabinetes do Ministério; Inspeção-Geral das Atividades em Saúde (IGAS), que tem como funções auditar, supervisionar e atuar disciplinarmente sobre os domínios da prestação dos cuidados de saúde, quer pertençam ao setor público quer se trate de entidades privadas ou do setor social; DirecçãoGeral da Saúde (DGS), que planifica, regula, dirige, coordena e supervisiona todas as atividades de promoção da saúde e prevenção de doença, assim como define as condições técnicas para a adequada prestação de cuidados de, sendo responsável pelos programas de saúde pública, qualidade na saúde, vigilância epidemiológica, estatísticas da saúde; Serviço de Intervenção nos Comportamentos Aditivos e nas Dependências (SICAD), que promove a redução do consumo de substâncias psicoativas, a prevenção dos comportamentos aditivos e a diminuição das dependências ${ }^{11}$.

\section{Setor Privado da Saúde em Portugal}

A iniciativa privada em Portugal tem destaque na cobertura e na qualidade dos serviços de saúde, quer numa perspectiva complementar ao SNS, quer como uma adicional.

Está contemplada na Lei de Bases da Saúde (1990) as convenções, celebração de acordos e contratação de serviços. Foram constituídas outras áreas de cooperação entre os setores público e privado: o programa de combate às listas de espera cirúrgicas - SIGIC (Sistema Integrado de Gestão de Inscritos para Cirurgia), a Rede Nacional de Cuidados Continuados Integrados (RNC$\mathrm{CI})$ e as Parcerias Público-Privadas (PPP) ${ }^{12}$.

Cerca de $40 \%$ da população portuguesa, beneficiária do SNS, tem em simultâneo um subsistema de saúde público (por exemplo: Assistência na Doença aos Servidores Civis do Estado - ADSE, Assistência na Doença aos Miltares - ADM, Subsistema de Saúde dos Serviços Sociais do Ministério da Justiça - SSMJ), privado ou um seguro de saúde (individual ou de grupo), direcionando para prestadores privados, o que reforça a relevância que o setor privado assume, presentemente, no quadro nacional da saúde. A oferta privada tem aumentado e é utilizada para superar lacunas ou fragilidades do setor público, como: a fraca cobertura; realização de meios complementares de diagnóstico e terapêutica; melhor nível de conforto nos serviços de hospitalização e; cuidados ligados à estética, entre outros $^{12}$.

\section{Serviço Nacional de Saúde (SNS)}

Nos anos 70 do século passado, ocorreram uma série de transformações na sociedade Portuguesa que originaram as marcantes reformas no Sistema de Saúde Português. Com o Decreto-Lei no 413/71 de 27 de setembro (Lei Orgânica do Ministério da Saúde) estabelece o novo Sistema Nacional de Saúde como um sistema unificado de saúde (componente pública e privada - incluindo as Misericórdias e outros privados ou sociais). Integrando os Serviços Médico-Sociais da Previdência, foi reconhecido o direito à saúde de todos os cidadãos, sendo pela primeira vez, identificado o papel do Estado como responsável pelas políticas de saúde e pela sua execução. Decorrente desta lei surge o Decreto-Lei no 414/71 onde se veem estabelecidas as carreiras profissionais do pessoal de saúde ${ }^{13}$.

Assim, a partir dos vários distritos, foram criadas duas organizações funcionais: os Centros e Saúde (Atenção Básica) e os Hospitais. O Ministério da Saúde reestruturou os serviços centrais, regionais, distritais e locais, passando a orientar, através das Direções Gerais de Saúde e dos Hospitais toda a política de saúde ${ }^{14}$. Esta reforma ficou conhecida como a reforma de Gonçalves Ferreira, sendo considerado o primeiro esboço do modelo público de saúde, contudo, só em 


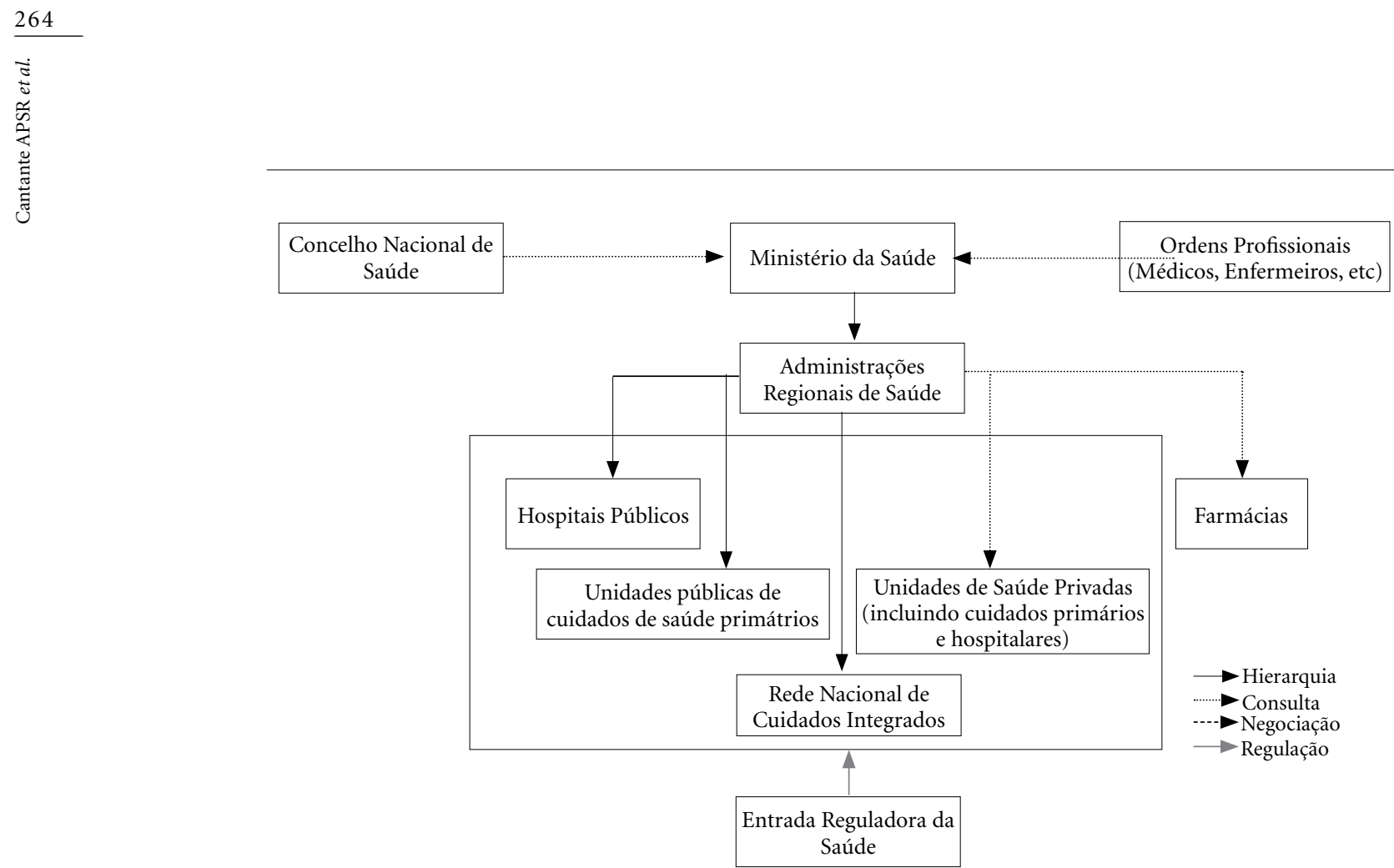

Figura 1. Sistema de Saúde Português.

Fonte: Simões et al. ${ }^{15}$.

1979 foi fundado o SNS, com as características principais que persistem até a atualidade.

Resultante da alteração da constituição Portuguesa (1976) que afirma que todos os cidadãos têm o direito de receber cuidados de saúde e da Lei da Saúde (1979), foi fundado o Serviço Nacional de Saúde no âmbito do Ministério dos Assuntos Sociais, para assegurar o direito à proteção da saúde, nos termos da Constituição. O SNS é gratuito e envolve todos os cuidados integrados de saúde, abrangendo a promoção e vigilância da saúde, a prevenção da doença, o diagnóstico, tratamento e a reabilitação médica e social. Tem autonomia administrativa e financeira com uma estrutura descentralizada central, regional e local, presta cuidados de saúde primários (atenção primária) e diferenciados (hospitais e instituições especializadas $)^{16}$.

Ao longo do percurso sofreu alterações e mantém os valores subjacentes à criação, mas enfrenta novos desafios. Os cidadãos têm direitos na saúde e a responsabilidade da saúde individual é, em primeira instância, dos cidadãos que tem o dever de defendê-la e promover. O SNS carateriza-se pela universalidade, prestação ou garan- tia de cuidados globais, tendência à gratuidade $\mathrm{e}$ equidade na acessibilidade aos cuidados.

Para atingir a missão, os serviços e entidades públicas prestadoras de cuidados de saúde, nomeadamente os Agrupamentos de Centros de Saúde - ACES (dependentes das Administrações Regionais de Saúde), os estabelecimentos hospitalares (independentemente da sua designação) e as Unidades Locais de Saúde - ULS, têm diretivas orientadoras, emanadas da Direção Geral de Saúde (DGS). Estas orientações decorrem do Plano Nacional de Saúde (PNS) com indicação de programas prioritários a serem trabalhados.

Na Figura 3 podemos observar o esquema do PNS extensão a 2020.

No nível local, em colaboração com as ARS e os ACES, e com a eventual participação dos municípios (divisões administrativas do território) são elaborados os planos de saúde locais, alinhados com o PNS e que atendem aos determinantes, da sua região, que influenciam a saúde individual, familiar e coletiva ${ }^{17}$.

A perspectiva dos cuidados de saúde primários concretiza-se, no SNS, por meio de uma rede de centros de saúde comunitários, com co- 


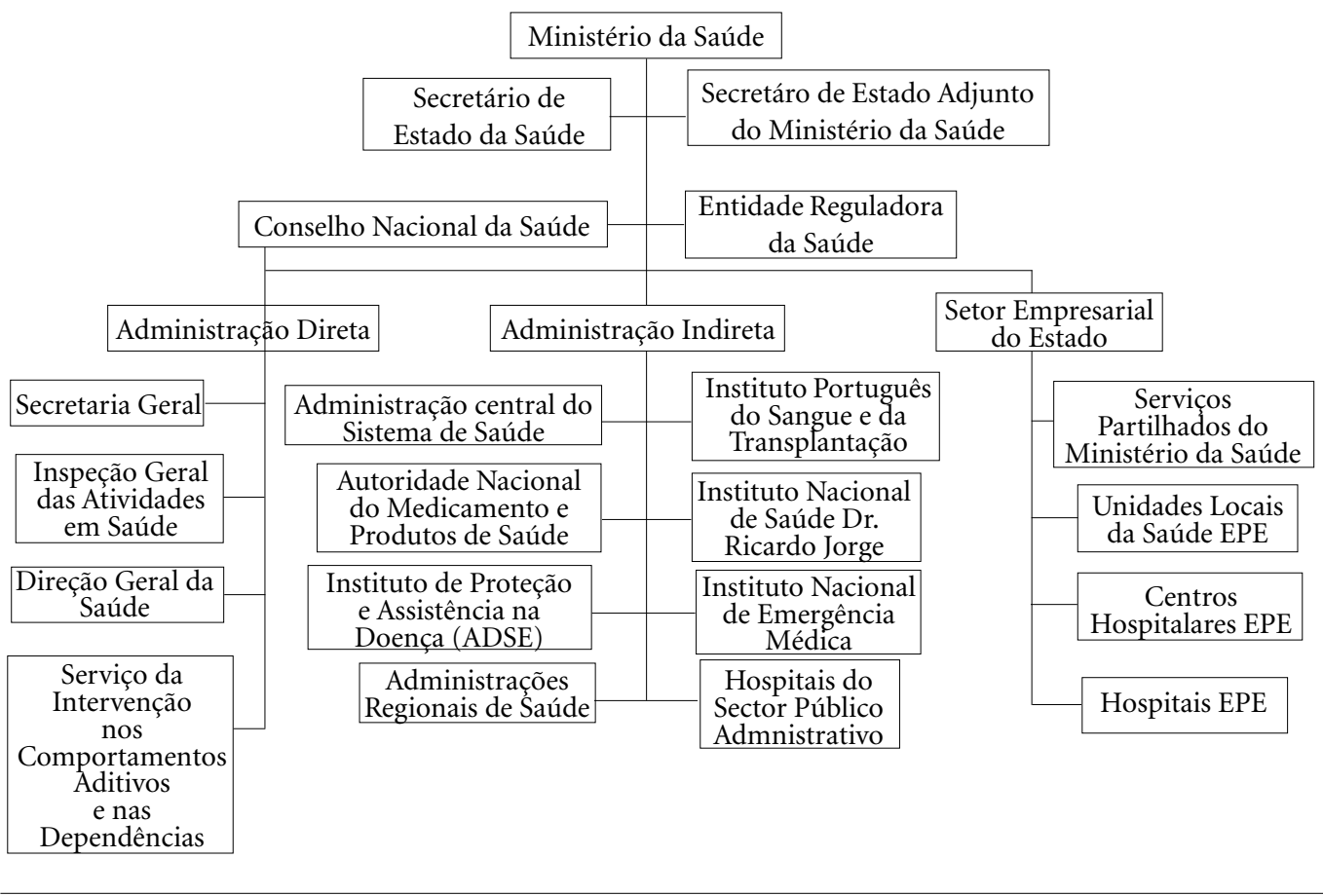

Figura 2. Organograma do Ministério da Saúde.

Fonte: Adaptado do SNS https://www.sns.gov.pt/institucional/entidades-de-saude/.

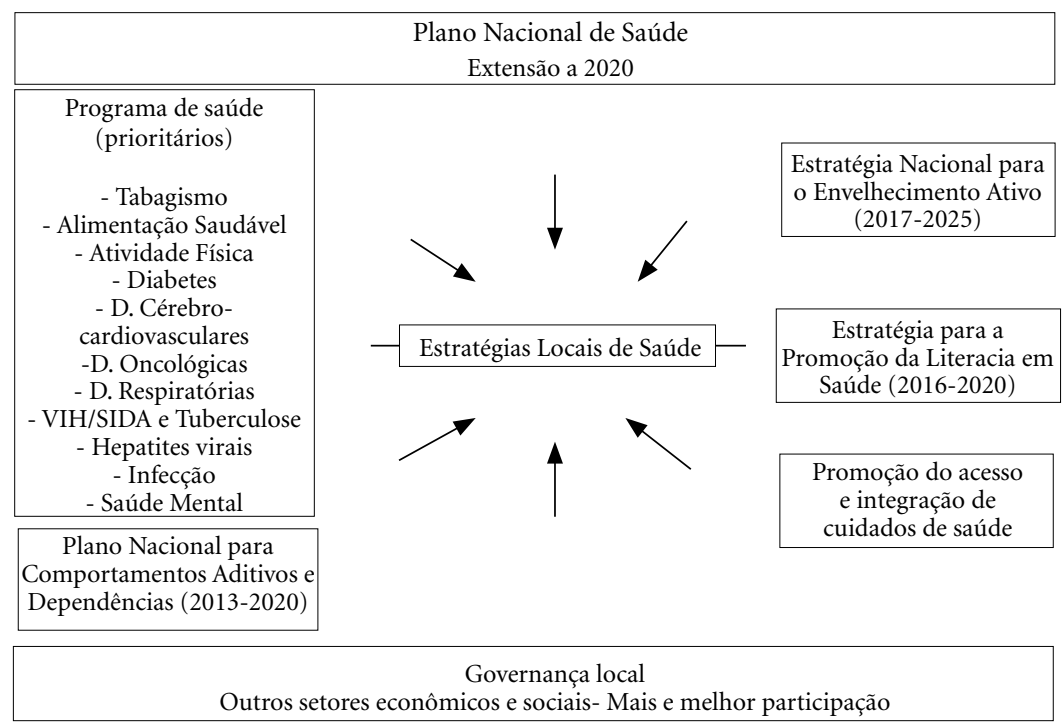

Figura 3. Esquema do PNS extensão a 2020.

Fonte: Adaptado do PNS https://www.dgs.pt/em-destaque/plano-nacional-de-saude-revisao-e-extensao-a-2020-aprovada-pelogoverno.aspx. 
bertura nacional e consequente de uma política de proximidade, são distribuídas por territórios residenciais. Os ACES são constituídos por várias unidades funcionais, que integram um ou mais centros de saúde ${ }^{11}$. Essas unidades funcionais são as Unidades de Saúde Familiar - USF, Unidades de Cuidados de Saúde Personalizados - UCSP, Unidades de Cuidados na Comunidade - UCC, Unidade de Saúde Pública - USP e Unidades de Recursos Assistenciais Partilhados - URAP.

Estas unidades básicas têm como missão a prestação de cuidados de saúde à população nas áreas de medicina geral e familiar, saúde infantil e juvenil, saúde materna, saúde pública e serviços sociais. Disponibilizam um conjunto de atividades diárias como consultas médicas e de Enfermagem, visitas domiciliárias, entre outras, que darão resposta às necessidades específicas da população que servem ${ }^{18}$.

\section{A Enfermagem em Portugal}

A Enfermagem é uma profissão que tem como objetivo prestar cuidados ao ser humano nos diversos contextos ao longo do ciclo de vida. O enfermeiro é um profissional detentor do curso de Enfermagem, legalmente reconhecido pelo Regulamento do Exercício Profissional do Enfermeiro - REPE, em seu Capítulo II, Artigo $4^{\circ}$ e do título profissional atribuído pela Ordem de Enfermagem - OE que lhe reconhece competências humanas, científicas e técnicas para a prestação de cuidados de Enfermagem ${ }^{19}$.

Segundo a OE, em dezembro de 2018 estavam inscritos 73.912 enfermeiros que exercem atividade nos diferentes contextos: Cuidados Gerais, 31.552; Prestação de Cuidados Especializados, 2.970; Assessoria/Consultadoria, 89; Ensino e Investigação, 357; Formação, 275; Gestão, 1.950; Outra, 564; Desconhecido, 36.155, sendo sempre na maioria do sexo feminino. Em relação à distribuição pelas especialidades: Enfermagem Comunitária, 2.869; Enfermagem de Reabilitação, 4.110; Enfermagem de Saúde Infantil e Pediátrica, 2.663; Enfermagem de Saúde Materna e Obstétrica, 2.917; Enfermagem de Saúde Mental e Psiquiátrica, 2.088; Enfermagem Médico-Cirúrgica, 4.035. São essencialmente Portugueses, embora haja enfermeiros de outras nacionalidades, com exceção da Oceânia todos os continentes estão representados, sendo o segundo país a Espanha e terceiro o Brasil. Quanto ao setor de atividade trabalham nos ACES - Cuidados de Saúde Primários, 7.836; nos cuidados diferenciados - Hospitais, 32.834; nas clínicas/laboratório,
1.051; outras instituições, 1.169; desconhecido, 30.503 e no ensino superior de Enfermagem, $519^{20}$.

A evolução ao longo dos séculos é notória, quer no nível da complexificação, quer no nível da dignificação do exercício profissional. O primeiro manual para enfermeiros surgiu em 1741 "A Postilla Religiosa e Arte de Enfermeiros", foi escrito por um enfermeiro (Frei Santiago). Nesse documento são apresentadas normas relacionadas com as funções dos enfermeiros, com duas dimensões: os conceitos de ciência, na época, e a sua experiência, mostrando uma perspectiva da Enfermagem do final da primeira metade do século XVIII. Passaram quase 200 anos desde que Florence Nightingale (1860) fundou a "Nova Enfermagem", com um novo paradigma e transformou-a numa profissão ${ }^{21}$.

Foi em 1881 que foi criada em Portugal a primeira escola para enfermeiros, nos Hospitais da Universidade de Coimbra e em 1896 surgiu outra escola na cidade do Porto, no Hospital Geral de Santo Antônio. Em 1899, foi fundado o International Council of Nurses (ICN), em 1900 surgiu o American Journal of Nursing e em 1901 foram criados os estatutos da Escola Profissional de Enfermeiros do H. Real de São José e Anexos, em Lisboa $^{22}$.

No século passado, em Portugal, foi profícuo para a Enfermagem. Ocorreram várias transformações que mais uma vez mudou o paradigma e o panorama nacional. Foram criadas várias escolas de Enfermagem públicas e privadas (muitas associadas a ordens religiosas) que formaram e habilitaram os enfermeiros para o exercício profissional. Em 1967 dá-se início à "sistematização dos saberes em Enfermagem, pelo uso da investigação e a identificação de uma dimensão intelectual dos cuidados de Enfermagem ... a disciplina começava a delinear-se como disciplina acadêmica e científica"22, passo significativo para a Enfermagem. Na década seguinte novas conquistas. Os enfermeiros passaram assumir cargos de diretores das escolas (até então eram os médicos), com autonomia técnica e administrativa.

Em 1973, a Federação dos Sindicatos Nacionais de Enfermagem, Associação Portuguesa de Enfermeiros, Associação Católica de Enfermeiros e Profissionais de Saúde, realizaram o $1^{\circ}$ Congresso Nacional de Enfermagem. No congresso foram abordadas temáticas precursoras que anteciparam o futuro da Enfermagem. Dele emergiram linhas condutoras que orientaram os anos vindouros como: a integração no Sistema Educativo Nacional, a necessidade de elaboração do 
estatuto profissional, e a discussão em torno da disciplina e profissão.

O reconhecimento acadêmico, dos seus vários níveis de formação, ocorre em 1988 com a publicação do Dec. Lei no 480, de 23 de dezembro, que regula a integração do ensino da Enfermagem no sistema educativo nacional, passando a ser ministrado em escolas superiores de Enfermagem. Em Portugal, o ensino de Enfermagem ao longo dos séculos sofreu imensas transformações, com o Decreto-Lei no $480 / 88$, de 23 de dezembro foi integrado no ensino superior. Dai decorreu uma série de alterações. Amendoeira ${ }^{23}$ resumiu a série de transformações que ocorreram (Figura 4).

$\mathrm{O}$ ensino de Enfermagem está estruturado em três ciclos de formação e de investigação: Formação Pré-Graduada - Licenciatura em ciclo único 4 anos; Mestrado em Ciências de Enfermagem (Instituto Ciências Biomédicas Abel Salazar - ICBAS em 1993/94) e Enfermagem (na Universidade Católica em 1991/92). Com Bolonha (Decreto-Lei no 74/2006, de 24 de março), possibilita a realização de dois tipos de $2^{\circ}$ ciclo na Universidade ou no ensino politécnico; Doutoramento em Ciências de Enfermagem (no ICBAS - Universidade do Porto, desde 2001; na Universidade de Lisboa em parceria com a Escola Superior de Enfermagem de Lisboa, desde 2004 e na Universidade Católica, por meio do Instituto de Ciências da Saúde Curso de Doutoramento em Enfermagem criado em 2004 e adequado a Bolonha em 2007. Há, em Portugal, 37 estabelecimentos de ensino públicos e privados, onde se pode frequentar o curso de Enfermagem.

Em paralelo, surgiram outros acontecimentos relevantes para a profissão como o REPE aprovado pelo Decreto-Lei no 161/96. Este regulamento é um instrumento jurídico aplicável em todos os contextos de atividade dos enfermeiros - público, privado ou em regime liberal. O exercício profissional deve ser desenvolvido “...com salvaguarda dos direitos e normas deontológicas específicos da Enfermagem como também por forma a proporcionar aos cidadãos deles carecidos cuidados de Enfermagem de qualidade" e "clarifica conceitos, procede à caracterização dos cuidados de Enfermagem, especifica a competência dos profissionais legalmente habilitados a prestá-los e define a responsabilidade, os direitos e os deveres dos mesmos profissionais, dissipando, assim, dúvidas e prevenindo equívocos por vezes suscitados não apenas no nível dos vários elementos integrantes das equipas de saúde, mas também junto da população em geral" ${ }^{19}$ (p.2).

Com a constante evolução da sociedade e para satisfazer níveis de saúde cada vez mais exigentes da população surge a necessidade de acesso a "padrões de cuidados de Enfermagem da mais elevada qualificação técnica, científica e ética”. Cria-se a OE e aprovando o respectivo Estatuto, pelo Decreto-Lei 104/98. A OE tem como propósito "promover a defesa da qualidade dos cuidados de Enfermagem prestados à população, bem como o desenvolvimento, a regulamentação e o controlo do exercício da profissão de enfermeiro, assegurando a observância das regras de ética e deontologia profissional"24(p.1741).

Consequentes dos contextos jurídico-institucionais do exercício profissional, os enfermeiros portugueses podem desempenhar atividade profissional em entidades do setor público, privado ou em regime liberal, do contexto dos cuidados de saúde primários ou diferenciados, nas áreas da prestação de cuidados, gestão e assessoria, ensino e investigação. O título profissional é atribuído pela Ordem dos Enfermeiros e só podem exercer, em Portugal, os enfermeiros detentores deste título.

O Conselho Internacional de Enfermeiros implementou a data de 12 de maio como o Dia Internacional de Enfermagem. Em Portugal também se comemora este dia, homenageando Florence Nightingale, enaltecendo os enfermeiros e divulgando o seu contributo para SNS e cuidados de saúde em geral. Em 2020, assinalam-se os 200 anos do nascimento deste ícone, e a OMS declarou o próximo ano como o Ano Internacional do Enfermeiro.

\section{Competências do Enfermeiro de Cuidados Gerais}

A competência do enfermeiro de cuidados gerais refere um nível de desempenho profissional demonstrador de uma aplicação efetiva do conhecimento e das capacidades, que lhe permitem o juízo clínico e a tomada de decisão ${ }^{25}$. O perfil competências surgiu de consensos sobre o "ICN Framework of Competencies for the Generalist Nurse" e desde 2003 orienta a prática de Enfermagem. Em 2015, os três domínios de competências - responsabilidade profissional, ética e legal; prestação e gestão de cuidados; desenvolvimento profissional - e as 96 competências foram reestruturadas para dar resposta ao seu processo de certificação (Figura 5).

O exercício das competências dos enfermeiros têm por base os seguintes pressupostos: (1) a relação interpessoal entre o enfermeiro e o cliente individual e/ou grupo - família ou comunidade -, com respeito pelos valores, crenças, projetos indi- 


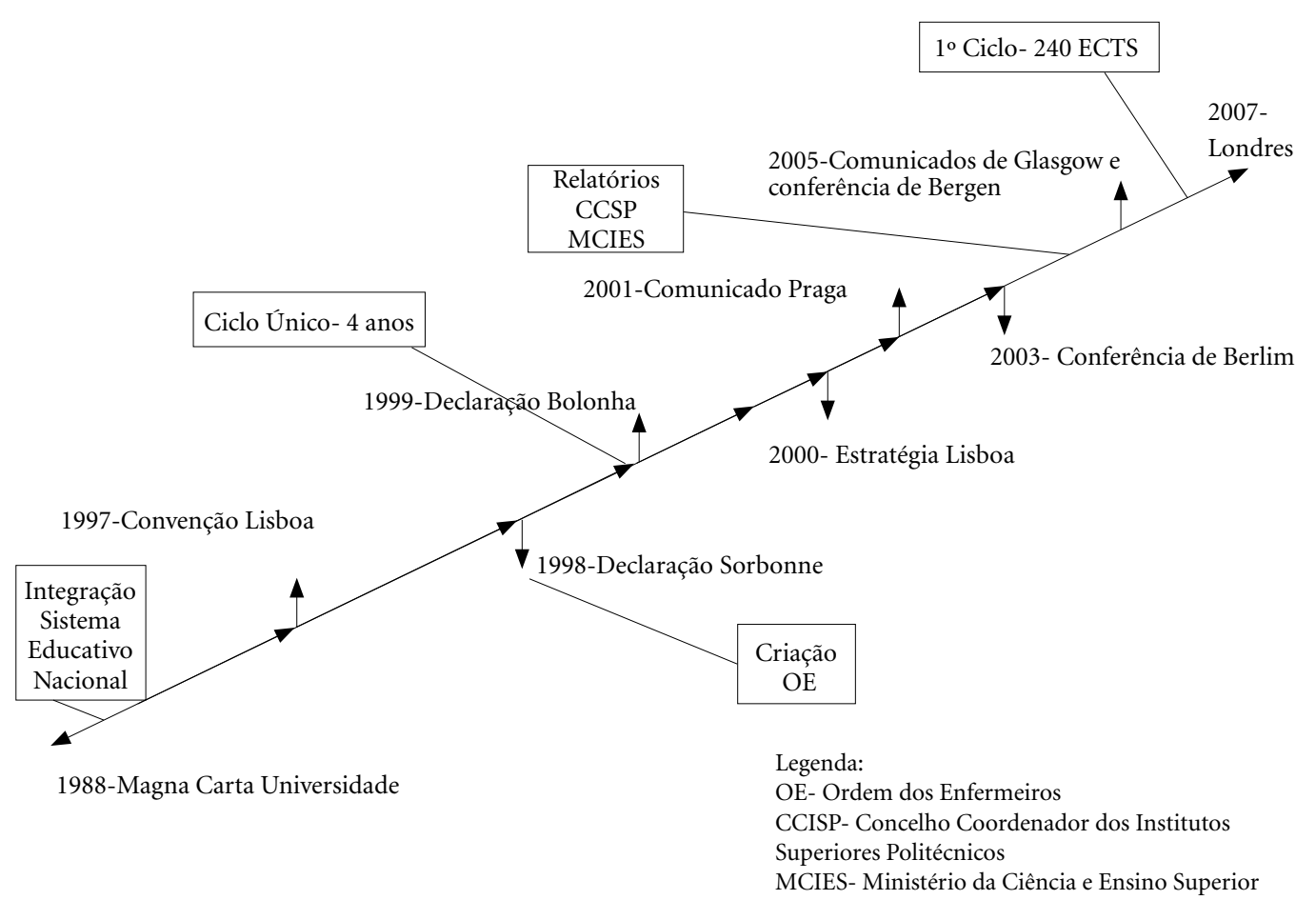

Figura 4. Estádios de Transição do Ensino de Enfermagem em Portugal a partir da década de 90.

Fonte: Amendoeira ${ }^{23}$.

viduais e capacidades, favorecendo o cuidado em parceria; (2) a tomada de decisão fundamentada em evidência científica, o juízo clínico fundamenta-se nas necessidades de cuidados individuais ou do grupo e nas intervenções de Enfermagem prescritas considerando a segurança dos cuidados e do cliente, a detecção precoce dos reais ou potencias diagnósticos procurando a resolução ou minimização das consequências; (3) humanistas, de respeito pela liberdade e dignidade humanas e pelos valores dos clientes e ainda, pelo respeito pelo preconizado no Código Deontológico e a regulamentação profissional, que estabelecem a boa prática $^{25,26}$.

$\mathrm{Na}$ concretização da meta, os enfermeiros portugueses adotam como conceito paradigmáticos: (1) a saúde enquanto o estado subjetivo e dinâmico da "condição individual, o controle do sofrimento, o bem-estar físico e o conforto emocional e espiritual" ${ }^{12}$ (p.8); (2) pessoa enquanto ser único e indivisível em permanente inter-relação com o ambiente e num processo intencional ou não, procura o equilíbrio, harmonia, o bem-estar e a concretização do seu projeto de saúde; (3) am- biente como o espaço onde se nasce, desenvolve e morre, a pessoa tem fator condicionante, inibidor ou facilitador, dos estilos de vida e consequentemente da saúde e; (4) cuidados de Enfermagem viabilizados pela relação terapêutica estabelecida entre enfermeiro-cliente e com foco no projeto de saúde individual ao longo do ciclo vital e na família enquanto alvo do processo de cuidados e parceira na potencialização da promoção da saúde.

Incluem dois tipos de intervenções de Enfermagem: as interdependentes, iniciadas por outros profissionais de saúde e as autónomas por enfermeiros, sendo da responsabilidade a prescrição e a implementação das ações de Enfermagem ${ }^{25}$.

\section{Competências do Enfermeiro Especialista}

O regulamento das Competências Comuns do Enfermeiro Especialista foi publicado pela primeira vez com o n ${ }^{\circ} 122$ em 2011, republicado com alterações em 2019 (Regulamento no 140). O regulamento clarifica os diferentes conceitos de competências (comuns, específicas e acrescidas, entre outos), assim como os diferentes do- 


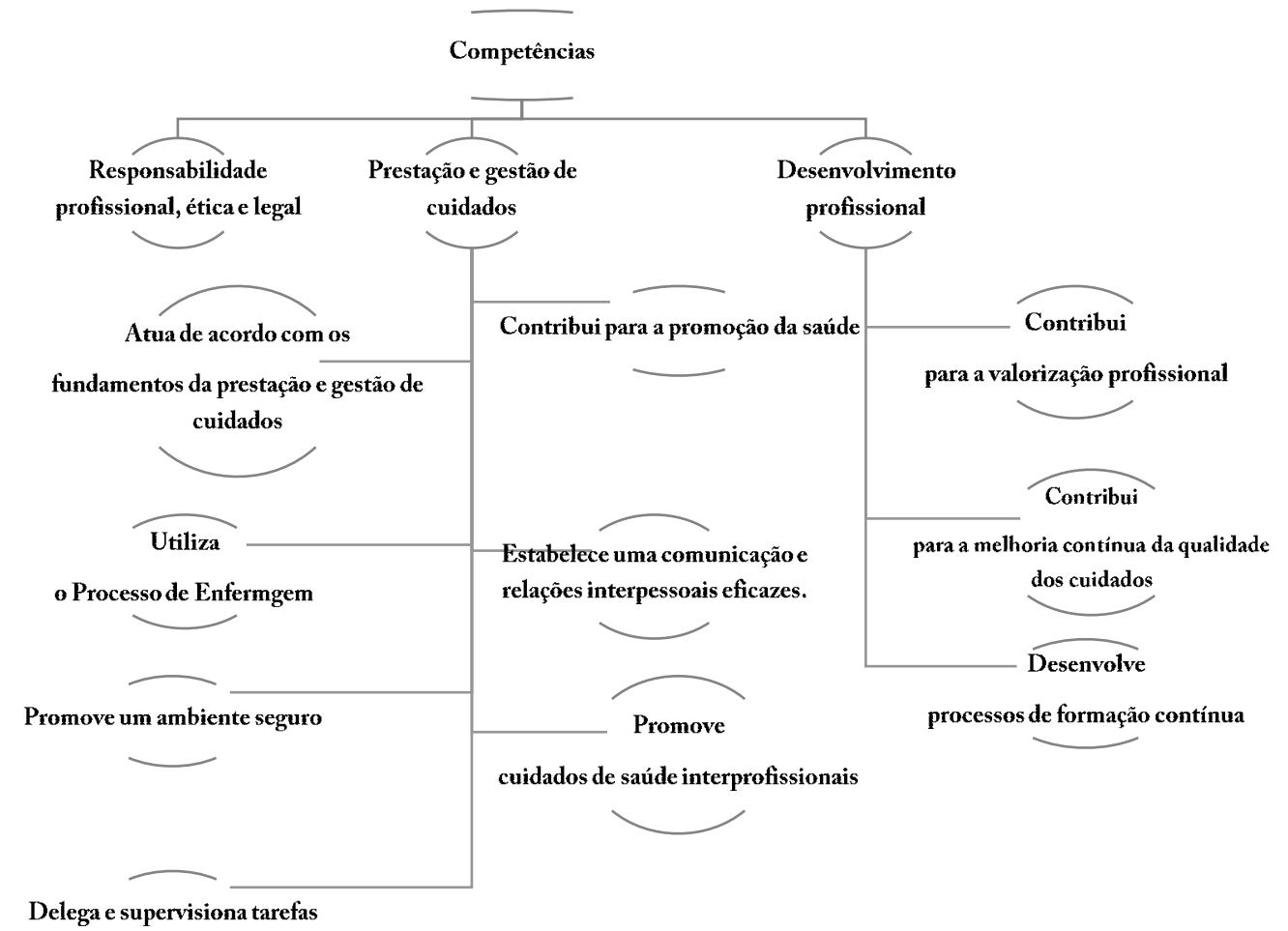

Figura 5. Síntese das Competências dos Enfermeiros de Cuidados Gerais.

Fonte: Adaptação dos autores.

mínios de competências comuns do enfermeiro especialista: responsabilidade, profissional, ética e legal (estão presentes no decorrer das suas intervenções); melhoria contínua da qualidade (no nível da governação clínica dinamiza e promove estratégias institucionais); gestão dos cuidados (organiza e estabelece redes e parcerias na equipe de Enfermagem e multiprofissional); desenvolvimento das aprendizagens profissionais (capacidade de desenvolvimento de autoconhecimento, assertividade nas relações terapêuticas e multiprofissionais em todos os contextos) ${ }^{27}$.

As diferentes especialidades em Enfermagem, com reconhecimento pela OE do título de enfermeiro especialista têm regulamentadas as competências específicas. Continuam reconhecidas pela $\mathrm{OE}$ as especialidades em Enfermagem de: Saúde Materna e Obstétrica (Regulamento no 321/2019); Saúde Infantil e Pediátrica (Regulamento no 422/2018); Saúde Mental e Psiquiátrica (Regulamento no 515/2018); Reabilitação (Regu- lamento no 322/2019); Médico-Cirúrgica (EMC - Regulamento no 429/2018) subdividida em EMC à Pessoa em Situação Paliativa, à Pessoa em Situação Crítica, EMC à Pessoa em Situação Perioperatória, EMC à Pessoa em Situação Crônica; Enfermagem Comunitária (Regulamento n ${ }^{\circ}$ 428/2018) subdividida na área de Enfermagem de Saúde Comunitária e Saúde Pública e na área de Enfermagem de Saúde Familiar.

Neste contexto foi publicado o Regulamento no 555/2017 (Regulamento de Certificação Individual de Competências) que certifica a "experiência profissional e os processos formativos dos Enfermeiros no seu todo, nos diferentes domínios de intervenção, que se visa certificar, de modo a permitir o posterior enquadramento numa situação de mais-valia profissional" ${ }^{128}$ (p.23633).

Assim os enfermeiros podem solicitar o título de Competência Avançada em: Gestão, Supervisão Clínica, Psicoterapia; Estomaterapia, e/ou a de Competência Diferenciada em: Supervisão 
Clínica, Enfermagem do Trabalho, Emergência Extra-Hospitalar, Estomaterapia, de acordo com "conjunto de conhecimentos, habilidades e atitudes, nos diversos domínios de intervenção, direcionado a atestar a formação, experiência ou qualificação do Enfermeiro numa área diferenciada, avançada e ou especializada, bem como a verificação de outras condições exigidas para o exercício da Enfermagem"28(p.23633). Igualmente, a OE, publicou os diferentes regulamentos de competências, nas referidas áreas, como orientação para a formação e reconhecimento das competências avançadas, no caso dos enfermeiros especialistas e diferenciadas para os enfermeiros de cuidados gerais.

Em Portugal, a Enfermagem tem um longo percurso de conquistas e evolução acompanhando o desenvolvimento científico e tecnológico, com o reconhecimento da capacidade de autorregulação, e na Classificação Nacional das Profissões (2008) está classificada como "Especialistas das Profissões Intelectuais e Científicas”. É uma profissão autônoma como preconiza a Lei de Bases do Sistema Educativo para o ensino universitário (Lei no 49/2005, de 30 de Agosto), que no artigo 11 diz "uma formação técnica que habilite para o exercício de atividades profissionais e culturais e fomente o desenvolvimento das capacidades de conceção, de inovação e de análise crítica" ${ }^{29}$ (p.5122). A formação pré-graduada em Enfermagem só pode ocorrer no âmbito do ensino superior no subsistema do ensino politécnico, enquanto que os mestrados podem ser oferecidos neste e no subsistema universitário, ficando o doutoramento em enfermagem exclusivamente neste último subsistema ${ }^{29}$.

A organização dos cuidados de enfermagem foca-se na eficiência e eficácia dos cuidados prestados sendo fulcral a existência de um quadro de referências e um sistema de qualidade para o exercício profissional e de registos que incorpore a sistematização da informação implícita no processo de enfermagem; uma dotação segura de cuidado e metodologias de trabalho; uma politica de formação contínua e uma avaliação da satisfação dos enfermeiros em função da qualidade do exercício profissional.

Os enfermeiros tiveram e têm uma participação na qualidade de vida dos cidadãos, e no sistema de saúde português. Perante a constante evolução e influência das novas tecnologias no cuidado de saúde e os emergentes desafios que se coloca, fica a reflexão, qual será o papel do enfermeiro nas gerações vindouras?

\section{Colaboradores}

APSR Cantante: concepção e desenho. HIVM Fernandes: concepção e desenho. MJ Teixeira: redação e revisão crítica. MA Frota: redação e revisão crítica. KMC Rolim: aprovação da versão a ser publicada. FHS Albuquerque: aprovação da versão a ser publicada. 


\section{Referências}

1. Portugal. Ministério da Saúde. Retrato da Saúde 2018 [livro na Internet]. [acessado 2018 Mar 21]. Lisboa: Ministério da Saúde; 2018. Disponível em: http:// www.insa.min-saude.pt/conheca-o-retrato-da-saude -em-portugal-2018/

2. Instituto Nacional de Estatística (INE). Conta Satélite da Saúde 2016-2019 [Internet]. 2019 [acessado 2018 Mar 21]. Disponível em: https://www.sns.gov.pt/noticias/2019/07/05/ine-divulga-conta-satelite-da-saude

3. Portugal. Ministério da Saúde. Serviços e Estabelecimentos do Serviço Nacional de Saúde [Internet]. 2018 [acessado 2018 Mar 21]. Disponível em: https://www. sns.gov.pt/sns/servico-nacional-de-saude/

4. Organisation for Economic Co-operation and Development (OECD), European Observatory on Health Systems and Policies. State of Health in the EU: Portugal: Perfil de Saúde do País 2017 [Internet]. Paris: OECD Publishing, Brussels: European Observatory on Health Systems and Policies; 2017. [acessado 2018 Mar 21]. Disponível em: http://dx.doi.org/10.1787/ 9789264285385-pt

5. Escoval A, Matos T, Ribeiro R, Santos ATL. Contratualização em Cuidados de Saúde Primários: Horizonte 2015/2. Fase 5: Relatório Final [relatório na Internet]. 2010 [acessado 2018 Mar 21]. Disponível em: http://portaisars.azurewebsites.net/wp-content/uploads/2017/01/contratualizacao_csp.pdf

6. World Health Organization (WHO). The world health report 2008: primary health care: now more than ever. Genebra: WHO; 2008

7. Andrade AC. A Regulação do Sistema de Saúde Português: Contribuição para um novo paradigma na prestação de cuidados de saúde. e-Pública [periódico na Internet]. 2016 [acessado 2018 Mar 21]; 3(3):201-221. Disponível em: http://www.scielo.mec.pt/pdf/epub/ v3n3/v3n3a10.pdf

8. Nunes E. Principais sistemas de saúde no mundo [Internet]. 2015 [acessado 2018 Mar 21]. Disponível em: http://www.rets.epsjv.fiocruz.br/sites/default/files/ arquivos/biblioteca/principais_sistemas_de_saude_ do_mundo.pdf

9. Portugal. Ministério da Saúde. Assembléia da República. Lei no 48, de 24 de agosto de 1990. Lei de Bases da Saúde [documento na Internet]. Diário da República; 1990. [acessado 2018 Mar 21]. Disponível em: https://dre.pt/pesquisa/-/search/574127/details/normal?p_p_auth=zt2dAYbd

10. Portugal. Ministério da Saúde. Decreto-Lei no 124, de 29 de dezembro de 2011. Aprova a Lei Orgânicado Ministério da Saúde [documento na Internet]. Diário da República; 2011. [acessado 2018 Mar 21]. Disponível em: https://data.dre.pt/eli/dec-lei/124/2011/12/29/p/ $\mathrm{dre} / \mathrm{pt} / \mathrm{html}$

11. Portugal. Ministério da Saúde. Decreto-Lei no 28, de 22 de fevereiro de 2008. Estabele o regime da criação, estruturação e funcionamento dos agrupamentos de centros de saúde do Serviço Nacional de Saúde [documento na Internet]. Diário da República; 2008. [acessado 2018 Mar 21]. Disponível em: https://dre. pt/application/conteudo/247675
12. Mateus A, Ramalho E, Oliveira H, Rodrigues H, Ferreira R. Setor Privado da Saúde em Portugal - Versão executiva [Internet]. AM\&A; 2017. [acessado 2018 Mar 21]. Disponível em: https://fronteirasxxi.pt/ wp-content/uploads/2018/02/Estudo-Sector_Privado_da_Sa\%C3\%BAde_em_Portugal.pdf

13. Portugal. Ministério da Saúde. Decreto-Lei no 414, de 22 de outubro de 1991. Visa definir o regime legal da carreira dos técnicos superiores de saúde dos serviços e estabelecimento do Ministério da Saúde e da Santa Casa Misericórdia de Lisboa [documento na Internet]. Diário da República; 1991. [acessado 2018 Mar 21]. Disponível em: https://data.dre.pt/eli/dec -lei/414/1991/10/22/p/dre/pt/html

14. Baganha MI, Ribeiro JS, Pires S. O Sector da Saúde em Portugal: funcionamento do sistema e caracterização sócio-profissional [Internet]. [acessado 2018 Mar 21]. Disponível em: https://www.ces.uc.pt/publicacoes/ oficina/182/182.pdf

15. Simões J, Augusto GF, Fronteira I, Hernández-Quevedo C. Portugal: Health system review. Health Systems in Transition [priódico na Internet]. 2017 [acessado 2018 Mar 21]; 19(2):16. Disponível em: http://www. euro.who.int/_data/assets/pdf_file/0007/337471/HiT-Portugal.pdf

16. Portugal. Ministério da Saúde. Lei no 56, de 15 de Setembro de 1979. Serviço Nacional de Saúde [documento na Internet]. Diário da República; 1979. [acessado 2018 Mar 21]. Disponível em: https://dre. pt/web/guest/pesquisa/-/search/369864/details/normal?_search_WAR_drefrontofficeportlet

17. Portugal. Ministério da Saúde. Plano Nacional de Saúde em revisão e extensão a 2020 [Internet]. 2015 [acessado 2018 Mar 21]. Disponível em: http://pns. dgs.pt/files/2015/06/Plano-Nacional-de-Saude-Revisao-e-Extensao-a-2020.pdf.pdf

18. Ministério da Saúde. Reforma dos Cuidados de Saúde Primários Plano Estratégico 2010-2011 [Internet]. 2007 [acessado 2018 Mar 21]. Disponível em: https:// www.sns.gov.pt/wp-content/uploads/2016/02/Plano -EstratÇgico-2010-2011-MCSP.pdf

19. Portugal. Dercreto-Lei no 161, 4 de setembro de 1996. Regulamento do exercício profissional dos enfermeiros - REPE [documento na Internet]. Diário da República; 1996. [acessado 2018 Mar 21]. Disponível em: https://www.ordemenfermeiros.pt/arquivo/AEnf ermagem/Documents/REPE.pdf

20. Ordem dos Enfermeiros (OE). Ensino de enfermagem e a sua potencial integração no subsistema universitário [Internet]. 2017 [acessado 2018 Mar 21] Disponível em: https://www.ordemenfermeiros.pt/ arquivo/membros/Documents/01.08.2017\%20-\%20 Tomada $\% 20$ de $\% 20$ posi $\%$ C3\%A7\%C3\%A3o- $\% 20$ Ensino\%20de\%20Enfermagem.pdf

21. Vieira M. Ser enfermeiro. Da compaixão à proficiência. $2^{\text {a }}$ ed. Lisboa: Universidade Católica Editora; 2009.

22. Amendoeira J. Profissões e estado: o conhecimento profissional em Enfermagem. In: Lima JA, Pereira HR, organizadores. Políticas públicas, conhecimento profissional: a educação e a Enfermagem em reestruturação. Porto: Pivpsic/Legis Editora; 2008. p. 112-130. 
23. Amendoeira J. Ensino de Enfermagem Perspectivas de Desenvolvimento. Pensar Enfermagem [periódico na Internet]. 2009 [acessado 2018 Mar 21]; 13(1):212. Disponível em: http://pensarEnfermagem.esel.pt/ files/2009_13_1_2-12\%282\%29.pdf

24. Portugal. Ministério da Saúde. Decreto-Lei no 104, de 21 de abril de 1998. Cria a Ordem dos Enfermeiros e aprova o respectivo Estatuto [documento na Internet]. Diário da República; 1998. [acessado 2018 Mar 21]. Disponível em: https://data.dre.pt/eli/dec -lei/104/1998/04/21/p/dre/pt/html

25. Portugal. Ordem dos Enfermeiros. Regulamento $n^{\circ}$ 190, de 6 de março de 2015. Regulamento do perfil de competências do enfermeiro de cuidados gerais [documento na Internet]. Diário da República; 2015. [acessado 2018 Mar 21]. Disponível em: https://dre. pt/application/conteudo/67058782

26. Queirós PJP, Vidinha TS, Almeida Filho AJ. Self-care: Orem's theoretical contribution to the nursing discipline and profession. Referência [periódico na Internet]. 2014 [acessado 2019 Ago 01]; IV(3):157163. Disponível em: https:// www.researchgate.net/ publication/269398253_Selfcare_Orems_theoretical_ contribution_to_the_Nursing_discipline_and_profession

27. Portugal. Ordem dos Enfermeiros. Regulamento $\mathrm{n}^{\circ}$ 140, de 18 de janeiro de 2019. Regulamento das Competências Comuns do Enfermeiro Especialista [documento na Internet]. Diário da República; 2019. [acessado 2019 Ago 01]. Disponível em: https://dre. pt/home/-/dre/119236195/details/maximized
28. Portugal. Ordem dos Enfermeiros. Regulamento $\mathrm{n}^{\circ}$ 555, de 20 de setembro de 2017. Regulamento de certificação individual de competências [documento na Internet]. Diário da República; 2017. [acessado 2019 Ago 01]. Disponível em: https://dre.pt/home/-/ dre/108315074/details/maximized

29. Portugal. Assembleia da República. Lei no 49 , de 30 de agosto de 2005. Segunda alteração à Lei de Bases do Sistema Educativo e primeira alteração à Lei de Bases do Financiamento do Ensino Superior [documento na Internet]. Diário da República; 2005. Disponível em: https://data.dre.pt/eli/lei/49/2005/08/30/p/dre/ $\mathrm{pt} / \mathrm{html}$
Artigo apresentado em 13/04/2019

Aprovado em 20/08/2019

Versão final apresentada em 20/09/2019 